\title{
Revolutionizing Telemedicine by Instilling H.265
}

\author{
Hafiz Suliman Munawar \\ Research Centre for Modeling and Simulation, National University of Sciences \& Technology (NUST, Pakistan) \\ Email: hafizsuliman91@gmail.com
}

\author{
Asad Ali Awan, Usama Khalid, Saba Munawar and Adnan Maqsood \\ Research Centre for Modeling and Simulation, National University of Sciences \& Technology (NUST, Pakistan) \\ Email:11beeaawan@seecs.edu.pk, usama_khalid@hotmail.co.uk, sabamunawar.iiui@gmail.com and \\ dradnanmaqsood@outlook.com
}

\begin{abstract}
The aim of telemedicine system is to diagnose patients remotely; this includes healthcare provision to patients in far flung areas. The process of remote diagnosis is mainly dependent on bandwidths which are absolutely essential in terms of communications via the networks. Such communications have to be carefully monitored, because randomly sending data across the network and loading of packets as direct stream of cameras would result in chocking the bandwidth. Hence it is extremely important to make sure that the data is compressed so that there is minimum usage of bandwidth. But this has to be done in a way that maintains the quality at its best. This retention of good quality along with minimizing the usage of data is done by means of compression the streams obtained through cameras and then decompressing the data at the other end. This purpose was previously achieved by H.264 codec. Our major target was up gradation of the existing codec by introducing the latest; H.265. The Libde265 (Decoder) and the x265 (encoder) libraries have been used for the purpose of developing the H.265 codec. The H265 is an advanced codec, having better quality and ability to achieve far better compression than its predecessor. It has been shown through the algorithm and coding of H.265 that is has better compressing ability and the quality is maintained during the transmission of videos. This is highly desirable for the field of telemedicine as it can make improvements in providing healthcare services by easing the transmission of data in the form of videos from the patient end to the doctor's end.
\end{abstract}

Index Terms-Telemedicine, H.265, Compression Standard, Minimizing Bandwidth, Stream Compression, Live Video Streaming, Network Delay Improvement, Diagnostic Camera

\section{INTRODUCTION}

The developed era has seen inflation in almost all sectors and the healthcare sector is no exception. The costs for healthcare have escalated a great deal and this has resulted in a chaotic situation for most of the population. There are fewer number of doctors and health service providers for people based in the less urbanized areas and hence they face a number of problems in seeking answers to their health related issues. The health experts and renowned doctors are not easily available in all facilities, this creates a gap in providing quality health services to all. There is a need to find solutions for these issues as the health of population is top priority for the prosperity of any country. One such solution has been served by the advent of telemedicine.

Telemedicine is among the emerging fields of science which makes use of information technology and telecommunications for provision of healthcare services to people situated in areas where the facilities are not available or updated. This is achieved by means of integrating the modern equipment and technological approaches. These new era inventions are integrated in hospital settings and clinics such that they become a tool of use for providing patients with care even in areas where the doctors cannot be physically present. This is playing an extremely important role in eliminating the obstacles that exist for the rural people in terms of meeting their basic healthcare needs. Also, telemedicine proves to play a vital role as life savior in emergency situations and during times of critical healthcare needs [1]

Advancements in technology had seen previous innovations that can be linked to telemedicine but it is the turn of the 20th century that saw the emergence of telemedicine as a result of continuous rise in the integration of telecommunication and information technology in various aspects of life [2]. Previously, it was the fields of business and education that were seen to be benefiting from the modern technology. But the recent years have seen a rise in the need of incorporating the new era equipment in the field of medicine. It is due to the positive effects of including such advancements in the field of medicine. These advancements have made it possible to make healthcare services available in deprived areas by means of technology. Patients present in areas with no facilities can be treated by doctors located far away due to telemedicine [3]. Communications between the two ends are achieved by well-formed systems that connect the medical staff end to the patient. It is not only the provision of care, but health informatics data, medical imaging and a lot other Healthcare services are provided to patients by means of telemedicine. This is further explained by the revolutionary effect of telemedicine which does to cut down the barriers and problems associated with healthcare in the remote areas [4]. The 
modern world does not consider distance as a barrier and with this feature being helpful in other areas of communication, the field of healthcare is also benefiting from it.

The earliest forms of telemedicine revolved around telephonic and radio contact between the doctor and the patient. This became advanced with the introduction of video-calling options and the advancements in the diagnostic methods have done to support these applications. The devices have also been upgraded and made better with time to ensure the best possible services to patients present in faraway localities.

\section{A. Advantages of Telemedicine}

There exist a number of advantages of telemedicine, including the efficient management of patients without the need of long travel time for the doctor or the patients. Moreover, time can be efficiently be managed on various fronts. There is the possibility of making reports and $\mathrm{x}$ ray being sent to the doctors without the need of sending the hardcopies via post which can be a sure cause of delay [5]. The existing advancements of technology have made sure that mankind enjoys gains in all kinds of communications without the barriers of time or distance. The same is the case with telemedicine, which is known to be revolutionizing the healthcare system. The most pronounced advantages of telemedicine include the reduction in the necessity of hospitalizing patients [6]. This helps in reducing the process of patient management, support and hospital expenses that are usually associated with chronic cases. Healthcare services are easily benefited from by means of video-calling options made available which has connected patients with physicians, specialists and various health centers. It supports the medical services which are 'Patient Centered'. Also beneficial for experts who can achieve mobility by means of dealing with various patients through video calls on phones and tablets. In addition, important in terms of security as the prisoners can be treated through video connections rather than going through the risky process of transporting prisoners [7]. Moreover, it supports a wide range of experts to be integrated as a teams involved in the patient care.

Along with the above mentioned advantages, the other positive outcomes of using telemedicine include the cheaper costs. The poor can greatly benefit from the expansion of telemedicine as the costs associated with the visit of far off cities and big hospitals are reduced in this way. Moreover, the remote area clinics do not charge very high and the presence of an online expert sitting back in the city can ensure that people receive the best support for their health issues at the lowest possible price [8]. In addition to reducing the needs of travel, cutting down the time wastage and reducing the cost, telemedicine has made possible for different doctors from the larger cities to be connected to the ones practicing in the less resourced areas and have a chance of coanalyzing the real time situations that occur in the lesser privileged parts of the country or the world at large. This ensures that health experts do not remain confined to the situations present in their own setting only, rather they can explore situations and issues existing elsewhere and have a chance to practice better this way. A profound effect can be generated in the field of healthcare by developing a team of experts working as a unit while still practicing in their own settings in the different parts of the city or country. This would although require proper training of doctors in terms of modern equipment and use of the technology involved in telemedicine. But in the long run, the benefits cannot be overlooked [9].

The most basic and simple layout followed in telemedicine is shown in Fig. 1. The most highlighted features of telemedicine are is easy usage, ability to record a healthcare session and saving the patient's record, videoconferencing platform that is of value to both, the patient and the healthcare provider [10]. Moreover, direct streaming of media can be achieved and mobile phones can be made of use in obtaining healthcare Although the use of mobile phones is not as smooth as the other systems, but the advancements are making sure that the modern world can do to move towards the use of mobile phones as a means of gaining access to healthcare services. The rapid increase in the number of software available for use on mobile phones is a great feature of modern technology and holds great promise for future benefits. This will help in ensuring that in near future, telemedicine can be revolutionized and access of healthcare could be made as easy as making a regular call.

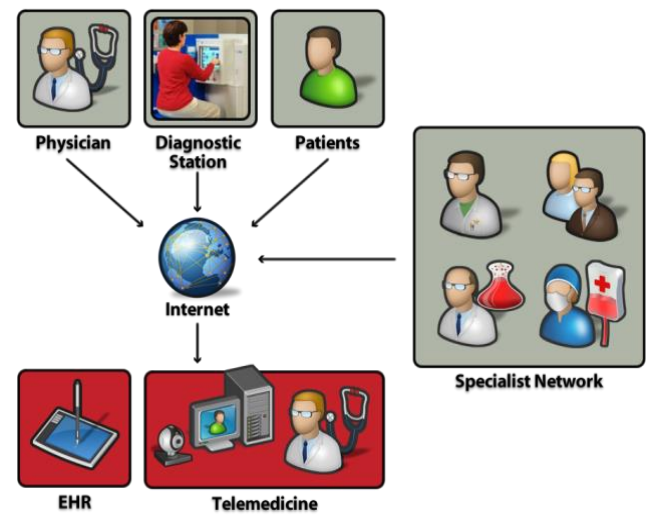

Fig.1. Concept of Telemedicine

\section{B. The current usage of telemedicine}

Telemedicine is being used worldwide in various areas of healthcare. Ranging from the basic areas of physiological checkups to diseases as severe as cancer and chronic heart disease patients in the lesser privileged areas are now being treated by doctors and specialists residing in big cities. All this has become possible due to the increasing acceptance of telemedicine as a helpful resource in the healthcare system. A number of chronic diseases are commonly found in the rural areas. These diseases include asthma, cardio-vascular diseases, pulmonary disorders, diabetes, hypertension etc [11]. Literature reveals that telemedicine has been in use for the treatment of patients suffering from such diseases and living in remote areas. The scope of telemedicine in 
regards to healthcare was established way back and by 2003, the use of telemedicine in the field of medicine became very much pronounced. Particularly for the chronic diseases, the presence of technology and the use of telemedicine is increasingly becoming common [12]. Major situations such as operations and cancer treatment are also being conducted by means of telemedicine approaches. Experts are available through online forums and can be approached for their say in critical matters such as major operations, management of chronic conditions and long term healthcare provision [13]. Apart from the treatment of chronic disease patients, the emergency departments of hospitals are also increasingly moving towards telemedicine. It has been found that the knowledge of expert doctors being just a call away is extremely comforting for the patients as well. Infections and the regularly occurring problems such as flu, nausea and other everyday problems are also now being sorted and dealt with by doctors who are providing their services through technology and are willing to divide their time between their clinics in metropolitan cities and the urban hospital settings. This being made possible without the need of time taking travelling is among the perks of telemedicine [14]. All these uses and the rapid acceptance of telemedicine reveal the future need and scope of the field. Patient care is extremely important and the need of experts in the remote areas can never be over-emphasized. Increasing the quality of life for people living in the underprivileged areas can only be achieved by efficiently managing the available resources, including the human resource. Since experts are rarely found in the remote areas, telemedicine can do to bridge the gap between doctors and patients residing in areas where the doctors cannot reach physically.

\section{EXISTING PROBLEMS}

The most prominent need of telemedicine is in terms of minimizing the requirement of time in providing healthcare. The distance and need of travelling is also reduced as the patient can be present in their own locality and the doctor can treat them through effective communications achieved by the telemedicine services. The most crucial point is to deal with the provision of effective communications. The patient's details and condition need to be sent to the doctor and visual contact should be available in order for the doctor to fully understand the conditions. If not for the videos, the doctor may not correctly understand the patient's condition and hence not have the necessary knowledge about the required treatment.

This is the major challenge in telemedicine. There is a very high demand of enhancing the video streaming quality and implement algorithms that can have a better network bandwidth. It is absolutely essential to have ample bandwidth available as video calling and medical imaging require a very high bandwidth as compared to simple mobile communications [15]. This is due to the nature of the medical information, particularly the $\mathrm{x}$-rays and the medical imaging results and reports that are needed to be sent to the doctor sitting miles away. They require a higher bandwidth and stronger systems and frameworks for their storage and transportation.

Two possible solutions for the existing problem of data transfer and sharing are either to increase the bandwidths of the wireless networks or to use the more sophisticated technology such as the video encoder which can utilize the available bandwidth. These possibilities have been explored on various occasions through research and interventional studies and it has been seen that there is a chance of improvement of the existing system through the development of better and more sophisticated frameworks that can do to match the newer technologies.

\section{A. Live Video Streaming}

Telemedicine essentially requires the use of live video streaming. This technology plays a very crucial in all sorts of telecommunications and tele-operations. But the problem lies in the delays associated with live streaming of videos. The main idea of this research is to understand the effects of delays caused during live streaming of videos and to find ways in which this delay can be cut down and effective systems could be incorporated [16]. This is absolutely necessary in case of treating patients who have an emergency situation and need medical attention rapidly. Delays in such cases can be highly dangerous [17]. Therefore, this study has explored the reasons of delays and has proposed measures that can cut down these delays and can improve the live video streaming.

\section{B. Live Streaming in Telemedicine}

It increases the convenience for the patients in terms of consultations and collaborating with the doctors and utilizes the technological workflow services to improve the healthcare provision. The rate of transmissions are reduced and the patient care is made better by making the follow-up procedures easy [18]. In addition to this, there is an enhancement in efficiency of communications, patient satisfaction through actively engaging them in their treatment. The most important feature is that these services can be used from smartphones too.

\section{Working of the Existing System}

The current codec used for video streaming in telemedicine is H.264 which is a video codec. The important features to consider while using any system are its quality, efficiency, and decoding capabilities. The following discussion outlines the main features of H.264

H.264 has the ability to be installed in many formats and is usually used in the MPEG-4 container format. This format makes use of the MP4 extension and also the Flash, 3GP in case of phones, QuickTime and the MPEG transport stream. The most widely used codec for compression encoded in H.264 is the Advanced Audio Coding (AAC) which happens to be an ISO standard [19]. Due to its establishment as a standard codec, H.264 has seen wide implementation and is known to deliver varying levels of quality and configurations according to the version used [20]. 
The compression parameters have a certain level of uniformity present for every codec. The H.264 makes use of varying Profiles while encoding a video. This can be explained by the algorithms that are used for compression by H.264. The major feature is the upgraded quality and complexity that makes it difficult to decode it [21].

The creation of bitstream is specified by the Profiles which determine the techniques that can be used. Example includes the Baseline profile which is among the most simple and widely used devices having lowpower. It does not make use of CABAC or the B-frames entropy encoding [22]. Good quality is still achieved but it is not at par with the streams produces by the Main or High Profiles that make use of both the techniques. As a rule, the Levels of specifications and the Profiles are extremely important during the production of devices as the videos have to be played accordingly [23]. Any wrong specification or Level will result in an incapability of playing the video on such devices. Whereas, computers utilize H.264 playback and all kinds of videos, even the advanced Profile are supported by most of the streaming encoding tools and these can have resolutions of $1080 \mathrm{p}$ and higher [24]. The major reason for the popularity of $\mathrm{x} 264$ lies in its wide array encoding parameters and the varying degree of optimizations that can be carried out.

\section{SWITCHING TOWARDS H.265}

Telemedicine is now being shifted to H.265 because of its ability to use lower bandwidth and compress the videos in a better way. Since the most important thing in the video-streaming is to reduce the data size so that files occupy less space and the overall bandwidth is reduced as to increase the rate of transmission, it is better to use H.265 as it provides all these specifications [25]. Therefore, the focus of the experts is now rapidly moving towards H.265, so as to match the needs of the healthcare facilities in a faster and more appropriate manner.

In order to provide the most efficacious compression, HEVC was created with the vision to be twice better than the H.264/AVC. The efficiency of compression can vary depending on the content that has to be delivered and the corresponding encoding. The HEVC is usually used for compressing the videos with twice the efficiency achieved by AVC. This is advantages for users and they can benefit in either of the two following ways:

- For the same level of quality in viewing, the HEVC can compress the video to half its original size/bit rate.

- The compressed file by HEVC provides much better quality as compared to the AVC compressed file of same size [26].

\section{A. Working Algorithm of H.265}

Transmitting End:

while(isConnected)

\{

Copyright @ 2017 MECS if(frame) \{

outputFrame = push_data_to_ecnoder(frame

tcp_Send_Data(outputFrame);

\})

push_data_to_ecnoder(frame)

\{

if (readPicture(frame))

inFrameCount++;

else

pic_in = NULL;

ecnode_data(frame);

encoderCounter $+=1$;

outFrameCount += numEncoded;

\section{$\underline{\text { Receiving End: }}$}

while(isConnected)

\{

outputFrame= decode_frame(Rcvframe);

\}

decode_frame(Rcvframe)

\{

push_data(Rcvframe);

more $=1$;

while (more)

\{

more $=0$;

img = decode(Rcvframe);

if (img)

\{

outputToRenderer(outputFrame);

\}

\}

outputToRenderer(outputFrame)

\{

memcpy(dest, outputframe->y_frame, (_width *

_height));

memcpy(dest + (_width * _height) $/ 2$, outputframe$>$ u_frame, (_width $*$ _height $) / \overline{4})$;

memcpy $($ dest + (_width * _height $)+$ (_width * _height $)$ /

4,outputframe->v_frame, (_width *_height) / 4);

outputsize $=($ _width $*$ _height $) / 2+($ _width $*$ _height $) /$ $2+$ (_width *_height);

\}

\section{H.265 VS H.264 COMPRESSION RATIO \& BANDWIDTH UTILIZATION}

The major comparison point lies in the enhanced rate of compression by the H.265, which has the ability to efficiently compress data and enable its transmission at a faster rate in comparison with H.264. This is mainly used in lowering the cost and the bandwidth required for transmission [27].

This implies that similar coding results in saving around $50 \%$ of the bit rate when H.265 is used. The efficiency of coding is higher than the H.264 and there are more tools available in H.265 for the reduction of the bit rate. Example: a size of $16 \times 16$ pixels is fixed in case of H.264 whereas the H.265 provides a wide range of 
coding unit, starting from $8 \times 8$ to $64 \times 64$ as the maximum [28].

Coding discrepancies are common with H.264 because of the independence of data sections in the H.264 coding [29]. Whereas the H.265 makes use of a determined code unit which is obtained by the regional information. The Fig. 2 clearly contrasts the quality enhancement and the delicacy of image quality maintained by the H.265 in comparison with H.264.

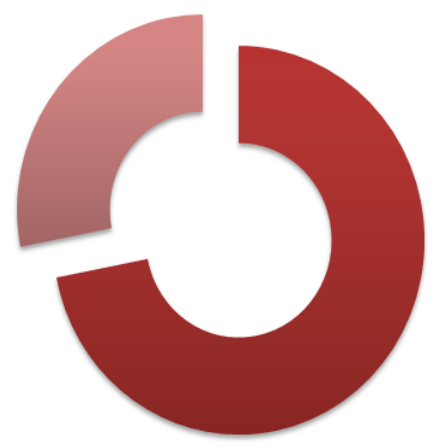

- H.264

H.265

Fig.2. Bandwidth Utilization of H. 264 vs H.265

For the same amount of data, the H.265 is capable of saving almost $70-80 \%$ of the bandwidth. The transfer of video is of high quality; $720 p$ at half the bandwidth required by H.264. This makes sure that the video is not disturbed owing to low speed of the internet (1-2Mbs).

\section{A. H.265 VS H.264 File Size}

Knowing that H.265 is much superior to the H.264, there is no doubt that the H.265 will now be more widely adopted and the field of telemedicine can greatly benefit from this adaptation. Fig. 3 clearly does to compare the rates of compression achieved by both the codecs. It is a great approach in maintaining quality with a reduced file size, this quality makes it a success over its predecessor.

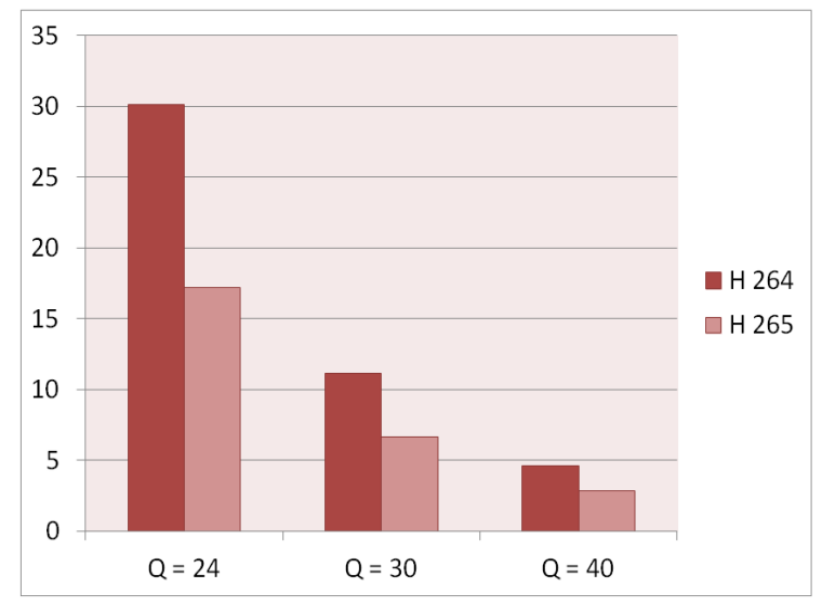

Fig.3. File size compression using H.264 Vs. H.265

B. What Makes HEVC Encode Videos with Twice the Efficiency of the Previous Standards?
The basic technique involved in compressing the videos is the 'motion compensated prediction'. In this technique, the pixels are encoded by means of referencing to different areas of the same frame or it can be any other frame. The HEVC can describe block size of up to $64 \times 64$ pixels, as compared to a mere $16 \times 16$ in case of H.264. The predicted blocks are coded in different sized blocks than the residual error. First, a prediction quad-tree is coded and then the encoder makes the decision about further encoding in terms of merging, skipping, inter or intra coding. The residual of these predictions is later coded and can have more depth than the quad-tree. This can make the $16 \times 16,8 \times 8$ or $4 \times 4$ transform to represent the error from $32 \times 32$ coding unit (CU) [30]. The HEVC is capable of encoding the motion vector with high precision and prediction of blocks can be achieved with lesser errors [31]. 35 intra-picture directions are present in comparison with mere 9 directions in case of H.264. Additionally, the newer methods of prediction are present in HEVC, such as the Adaptive Motion Vector Prediction as shown in fig. 4. The deblocking filters of HEVC are also quite improved and Additional filter for reducing the artifacts at the edges is present; the Sample Adaptive Offset. All these specifications make H.265 a much better codec than its predecessor.

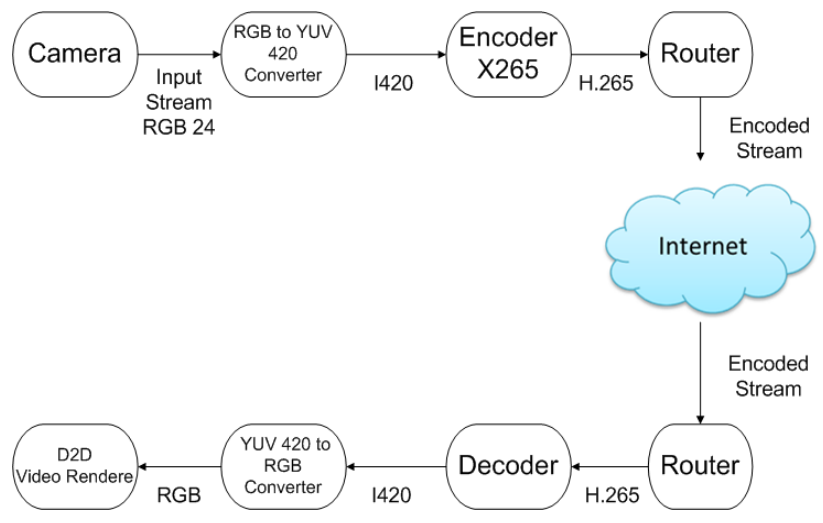

Fig.4. Backend Workflow of Proposed System

\section{CONCLUSION}

In conclusion, it can be said that H.265 is a much better codec in terms of utilization of band width and quality. Since the comparison between H.264 and H.265 has clearly showed that there is a much better quality achieved in video streaming when using H.265, it has become clear that there is a need to switch towards H.265 systems in telemedicine. Moreover, the data compressibility and decoding at the other end are also far better when H.265 is uses. It has been seen that with huge amounts of data, H.264 gets choked and it results in an inefficient transfer of data from patient end to the physician's end. Therefore, it is correct to say that the advancements in the codec and the development of H.265 is a major leap in making telemedicine more effective and easy access of healthcare can be provided to patients by opting for H.265. Incorporation of this system is likely to 
be a positive approach for the field of telemedicine. Moreover, with the rapidly evolving systems and needs of the modern society, it is necessary to keep an eye on the best possible technological solutions that can be used in the field of medicine for the benefit of mankind. This field is on the rise since more than 20 years and the modern era and continuous improvement in technology implies that the scope is still large and there is still a lot to be explored. Working in the current time of the century, we have proposed a framework that is relatively newer and better in terms of meeting the needs of data transfer and communication in the field of medicine. There is a chance that this may be changed in the future by better frameworks that evolve as a result of newer technology. Hence, the overall aim of proposing a modern and more sophisticated framework has been worked upon in this study. This could be further refined and made better in near future.

\section{FUTURE ENHANCEMENTS}

As the major goal of technology in the area of medicine is to enhance the quality of care to the patients, it is absolutely essential to move towards better technology. Future enhancements lie in finding better technological solutions that can help in making video streaming more efficient and easy. Such enhancements will help in refining the systems and also make the healthcare system more powerful and far-reaching. The time required to access medical help can be cut down by adopting better systems for telemedicine in future. Developing new measures and technological frameworks is an absolute necessity in this era. With the increase in the disease burden of the society, it will be worthwhile to have measures in place that do to ensure that even people living in relatively less privileged areas can get access to doctors and hospitals in a short span of time, without the need of travelling to far off cities. The framework proposed here can be further refined to meet these needs. The future holds great promise for the further expansion of the field of telemedicine and with that, possibly the systems and frameworks currently under consideration are likely to be refined and renewed over time.

\section{REFERENCES}

[1] World Health Organization. (2010). Telemedicine: Opportunities and Developments in Member States. Report on the Second Global Survey on E-Health. World Health Organization.

[2] Bensink, M., Hailey, D., \& Wootton, R. (2006). A systematic review of successes and failures in home telehealth: preliminary results. Journal of Telemedicine and Telecare, 12(suppl 3), 8-16.

[3] Wootton, R. (2012). Twenty years of telemedicine in chronic disease management-an evidence synthesis. Journal of telemedicine and telecare, 18(4), 211-220.

[4] Whitten, P. S., Mair, F. S., Haycox, A., May, C. R., Williams, T. L., \& Hellmich, S. (2002). Systematic review of cost effectiveness studies of telemedicine interventions. Bmj, 324(7351), 1434-1437.
[5] Rajan, B., Seidmann, A., \& Friedman, D. (2017, January). Managing Migraine via Telemedicine: Clinical Effectiveness and Process Implications. In Proceedings of the 50th Hawaii International Conference on System Sciences.

[6] Sood, A., Granick, M. S., Trial, C., Lano, J., Palmier, S., Ribal, E., \& Téot, L. (2016). The role of telemedicine in wound care: A review and analysis of a database of 5,795 patients from a mobile wound-healing center in Languedoc-Roussillon, France. Plastic and Reconstructive Surgery, 138(3S), 248S-256S.

[7] Weinstein, R. S., Lopez, A. M., Joseph, B. A., Erps, K. A., Holcomb, M., Barker, G. P., \& Krupinski, E. A. (2014). Telemedicine, telehealth, and mobile health applications that work: opportunities and barriers. The American journal of medicine, 127(3), 183-187.

[8] Yoo, B. K., Kim, M., Sasaki, T., Melnikow, J., \& Marcin, J. P. (2016). Economic evaluation of telemedicine for patients in ICUs. Critical care medicine, 44(2), 265-274.

[9] Ayatollahi, H., Sarabi, F. Z. P., \& Langarizadeh, M. (2015). Clinicians' knowledge and perception of telemedicine technology. Perspectives in health information management, 12(Fall).

[10] Amadi-Obi, A., Gilligan, P., Owens, N., \& O'Donnell, C. (2014). Telemedicine in pre-hospital care: a review of telemedicine applications in the pre-hospital environment. International journal of emergency medicine, 7(1), 1.

[11] Gibson, J., Lightbody, E., McLoughlin, A., McAdam, J., Gibson, A., Day, E., ... \& Ford, G. A. (2016). 'It was like he was in the room with us': patients' and carers' perspectives of telemedicine in acute stroke. Health Expectations, 19(1), 98-111.

[12] Sims, J. M. (2016). Communities of practice: telemedicine and online medical communities. Technological Forecasting and Social Change.

[13] Gamble, J.E., Savage, G.T. and Icenogle, M.L., 2004. Value-chain analysis of a rural health program: toward understanding the cost benefit of telemedicine applications. Hospital topics, 82(1), p.10.

[14] Wagner, K. L., \& Schafer, D. J. (2016). Using Telemedicine to Provide Safe Care When Birth Occurs in the Emergency Department. Journal of Obstetric, Gynecologic \& Neonatal Nursing, 45(3), S11-S12.

[15] Xiang, W., Wang, G., Pickering, M., \& Zhang, Y. (2016). Big video data for light-field-based 3D telemedicine. IEEE Network, 30(3), 30-38.

[16] Panayides, A., Eleftheriou, I., \& Pantziaris, M. (2013). Open-source telemedicine platform for wireless medical video communication. International journal of telemedicine and applications, 2013.

[17] Durner, G., Durner, J., Dunsche, H., Walle, E., Kurzreuther, R., \& Handschu, R. (2016). 24/7 Live Stream Telemedicine Home Treatment Service for Parkinson's Disease Patients. Movement Disorders Clinical Practice.

[18] Chakareski, J. (2013). Adaptive multiview video streaming: challenges and opportunities. IEEE Communications Magazine, 51(5), 94-100.

[19] Seeling, P., \& Reisslein, M. (2012). Video transport evaluation with H. 264 video traces. IEEE Communications Surveys \& Tutorials, 14(4), 1142-1165.

[20] Stutz, T., \& Uhl, A. (2012). A survey of h. 264 avc/svc encryption. IEEE Transactions on circuits and systems for video technology, 22(3), 325-339.

[21] Panayides, A., Antoniou, Z. C., Mylonas, Y., Pattichis, M. S., Pitsillides, A., \& Pattichis, C. S. (2013). High- 
resolution, low-delay, and error-resilient medical ultrasound video communication using H. 264/AVC over mobile WiMAX networks. IEEE journal of biomedical and health informatics, 17(3), 619-628.

[22] Xu, D., Wang, R., \& Wang, J. (2012). Prediction mode modulated data-hiding algorithm for H. 264/AVC. Journal of Real-Time Image Processing, 7(4), 205-214.

[23] Kim, H., Rhee, C. E., \& Lee, H. J. (2015). An Effective Combination of Power Scaling for H. 264/AVC Compression. IEEE Transactions on Very Large Scale Integration (VLSI) Systems, 23(11), 2685-2689.

[24] Wang, Y., Li, P., Zhang, P., Zhang, C., \& Cong, J. (2013, May). Memory partitioning for multidimensional arrays in high-level synthesis. In Proceedings of the 50th Annual Design Automation Conference (p. 12). ACM.

[25] Quinlan, J. J., Zahran, A. H., \& Sreenan, C. J. (2016, May). Datasets for AVC (H. 264) and HEVC (H. 265) evaluation of dynamic adaptive streaming over HTTP (DASH). In Proceedings of the 7th International Conference on Multimedia Systems (p. 51). ACM.

[26] Torres, A., Pinol, P., Calafate, C. T., Cano, J. C., \& Manzoni, P. (2014, April). Evaluating h. 265 real-time video flooding quality in highway V2V environments. In 2014 IEEE Wireless Communications and Networking Conference (WCNC) (pp. 2716-2721). IEEE.

[27] Asaduzzaman, A., \& Gunasekara, G. H. (2014). Power and performance analysis of multimedia applications running on low-power devices by cache modeling. Multimedia tools and applications, 72(1), 207230.

[28] Grois, D., Marpe, D., Mulayoff, A., Itzhaky, B., \& Hadar, O. (2013, December). Performance comparison of $h$. 265/mpeg-hevc, vp9, and h. 264/mpeg-avc encoders. In Picture Coding Symposium (PCS), 2013 (pp. 394-397). IEEE.

[29] Wiegand, T., Sullivan, G. J., Bjontegaard, G., \& Luthra, A. (2003). Overview of the H. 264/AVC video coding standard. IEEE Transactions on circuits and systems for video technology, 13(7), 560-576.

[30] Santos, H., Rosário, D., Cerqueira, E., Camargo, J., Schimuneck, M., Nobre, J., \& Both, C. (2016, October). A Comparative Analysis of H. 264 and H. 265 with Different Bitrates for on Demand Video Streaming. In Proceedings of the 9th Latin America Networking Conference (pp. 53-58). ACM.

[31] Ohm, J. R., Sullivan, G. J., Sze, V., Wiegand, T., \& Budagavi, M. (2016). Introduction to the Special Issue on HEVC Extensions and Efficient HEVC Implementations. IEEE Transactions on Circuits and Systems for Video Technology, 26(1), 1-3.

\section{Authors' Profiles}

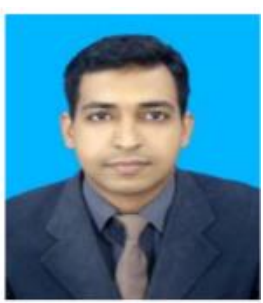

Hafiz Suliman Munawar received his B.Sc Engineering degree in 2014 from UET Taxila, Pakistan. Currently he is MS research student at Research Center for Modeling and Simulation, National University of Sciences and Technology (NUST), Pakistan. With a competent interest in multidisciplinary studies.

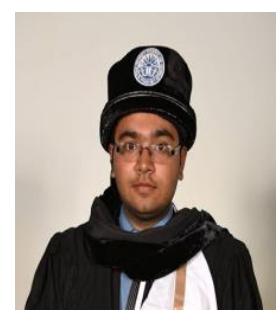

Asad Ali Awan is working as a Software Development Engineer in Telesehat Pvt. Ltd. Pakistan. He has been associated with NUST since 5 years and completed his BS Electrical Engineering from National University of Sciences and Technology (NUST), Pakistan in 2015. and also worked as a Research Assistant in NUST Pakistan. $\mathrm{He}$ has a sharp understanding of video encoding and Telemedicine system.

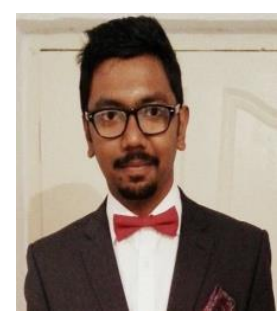

Usama Khalid is working as a Research Assistant in Telesehat Pvt. Ltd. Pakistan and received his BS (Software Engineering) degree from COMSATS Institute of Information Technology, Pakistan in 2016. He has been working on TeleDiagnosis to optimize its performance.

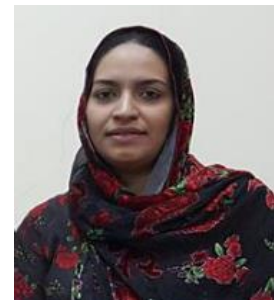

Saba Munawar is a $\mathrm{PhD}$ Research Scholar at Research Center for Modeling and Simulation, National University of Sciences and Technology (NUST) in Bioinformatics. She has a sound knowledge of Telemedicine equipment, remotely diagnostic camera's and formal procedures development

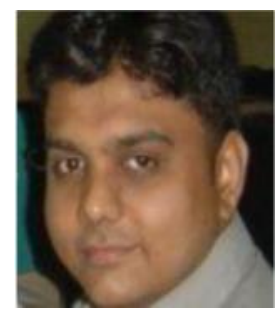

Dr. Adnan Maqsood is working as Assistant Professor at Research Center for Modeling and Simulation, National University of Sciences and Technology (NUST), Pakistan, since 2012. He received his Bachelor degree in Aerospace Engineering from NUST, Pakistan in 2005 and $\mathrm{PhD}$ from Nanyang Technological University (NTU) 
How to cite this paper: Hafiz Suliman Munawar, Asad Ali Awan, Usama Khalid, Saba Munawar, Adnan Maqsood,"Revolutionizing Telemedicine by Instilling H.265", International Journal of Image, Graphics and Signal Processing(IJIGSP), Vol.9, No.5, pp.20-27, 2017.DOI: 10.5815/ijigsp.2017.05.03 